\title{
Producing OAM Information Carriers using Micro-structured Spiral Phase Plates
}

\author{
Edgars Stegenburgs, ${ }^{1}$ Andrea Bertoncini, ${ }^{2}$ Abderrahmen Trichili, ${ }^{1}$ Mohd Sharizal \\ Alias, ${ }^{1}$ Tien Khee Ng, ${ }^{1}$ Mohamed-Slim Alouini, ${ }^{1}$ Carlo Liberale, ${ }^{2}$ and Boon S. Ooi ${ }^{1}{ }^{*}$ \\ ${ }^{1}$ Computer, Electrical and Mathematical Science and Engineering (CEMSE) Division \\ King Abdullah University of Science and Technology (KAUST), Thuwal 23955-6900, Saudi Arabia \\ ${ }^{2}$ Biological and Environmental Science and Engineering (BESE) Division \\ King Abdullah University of Science and Technology (KAUST), Thuwal 23955-6900, Saudi Arabia \\ *Author e-mail address: boon.ooi@kaust.edu.sa \\ https://photonics.kaust.edu.sa
}

\begin{abstract}
We report on small foot-print spiral phase plates for orbital angular momentum (OAM) light beam generation used in free space communication. A modal decomposition process confirms high purity of the generated beams at 980-nm wavelength. (C) 2019 The Author(s)
\end{abstract}

OCIS codes: 050.4865 Optical vortices, 060.4230 Multiplexing, 110.6895 Three-dimensional lithography

\section{Introduction}

Following the seminal paper of Gibson and co-workers, communicating using OAM modes have recently received a considerable attention [1]. Various technologies have been demonstrated to generate OAMs including spatial light modulators, q-plates, spiral phase plates (SPPs) and meta-surfaces [2]. However, moving beyond laboratory test benches and practically implement OAM-based communication systems requires new mode generation techniques [3]. OAM generation devices with small footprints are of high interest to many fields, including optical wireless communication, optical fiber connections, and on-chip-scale interconnects.

In this paper, we design and fabricate 3-D micro-scale spiral phase plates that produce pure OAM beams proven to deliver high-bit-rate signals with high fidelity efficiently. This generation method opens new opportunities for OAM-based integrated optical communication systems.

\section{SPP Fabrication and the Experimental Setup}

The design of an SPP for an OAM with a topological charge $\ell$ is performed according to $H=\lambda /\left(n-n_{0}\right)$, where $H$ is the height difference of SPP helical surface, $\lambda$ is the wavelength, and $n$ is the refractive index of the SPP material while $n_{0}$ is the refractive index of the medium. The 3-D models of four different topological charge SPPs are shown in Fig. 1(a).

(a)
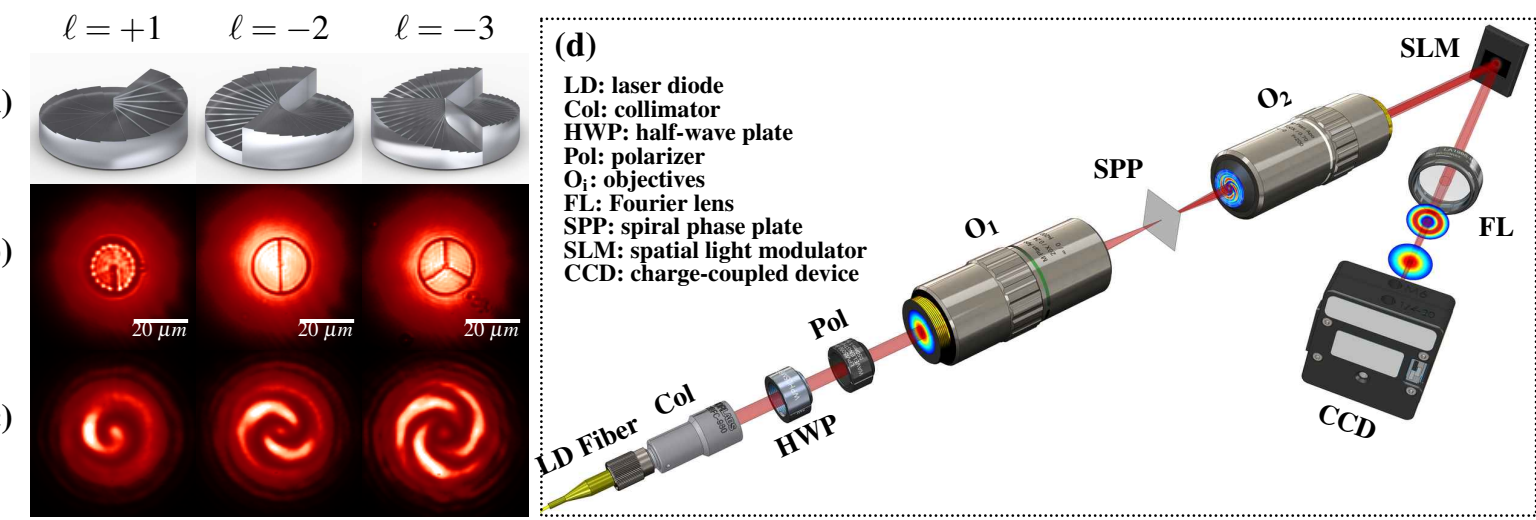

Fig. 1. (a) 3D models of SPP structures. (b) Micro-scale SPPs illuminated by 980-nm wavelength light. (c) The experimental single-beam interferograms. (d) Schematic of the experimental setup.

SPPs with $20 \mu \mathrm{m}$ diameters are fabricated with a commercially available two-photon absorption 3D direct laser writing tool (Nanoscribe Photonics Professional GT). The fabricated SPPs and the corresponding interferogram 
profiles are depicted in Fig. 1(b,c). The experimental setup for the produced OAM beams purity measurement is shown in Fig. 1(d). The purity of the OAM generated modes is verified using the modal decomposition method incorporating a CCD camera fixed at the Fourier plane of a spatial light modulator [4]. The SLM (Holoeye PLUTO2 NIR-015) is loaded with digital holograms programmed using a complex amplitude modulation technique [5], for topological charges ranging from $\ell_{S L M}=-10$ to 10 .

\section{OAM Mode Purity and Optical Communication Performance}

The decomposition graphs are depicted in Fig. 2(a). The modes are found of high purity with minimum crosstalk with the neighboring modes that is due to slight misalignment of the setup when moving the glass substrate manually. The resulting intensity profiles resulting from the decomposition are shown in Fig. 2(b).
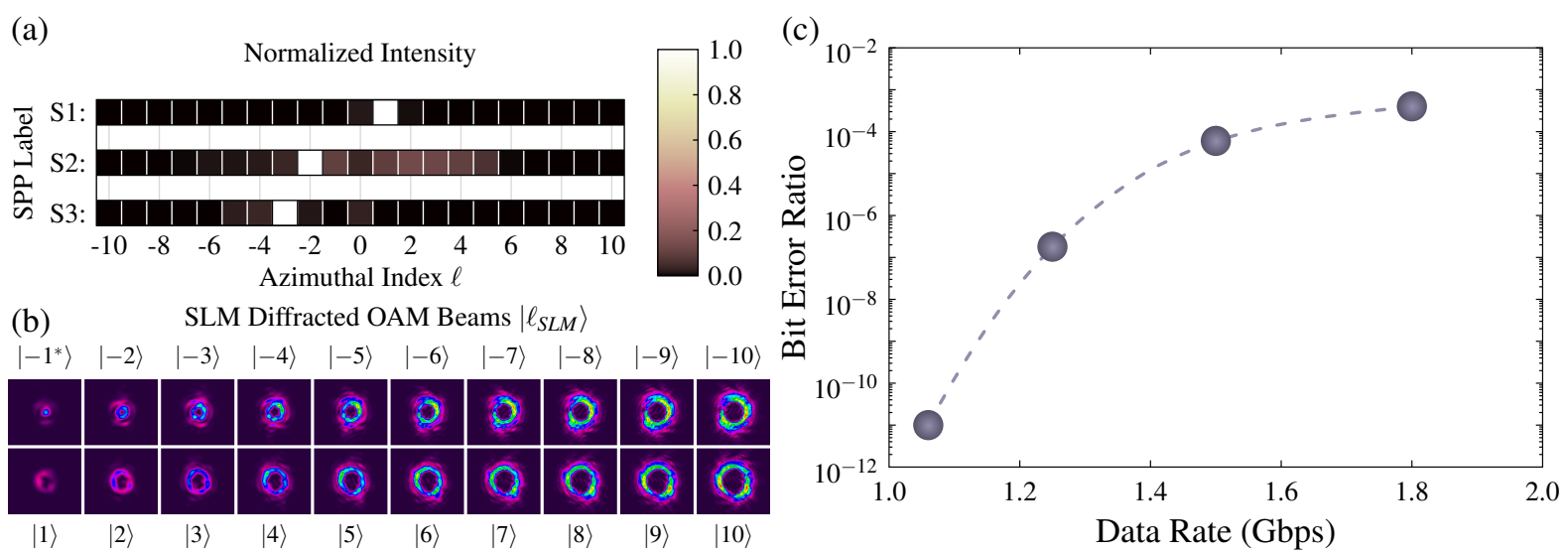

Fig. 2. (a) The OAM mode purity measurements for different SPPs. (b) Modal decomposition of 20 $\mu \mathrm{m}$ SPP with $\ell=+1$. (c) BER performance vs. signal data rate.

To assess the potential of using the SPPs in communication systems, we replaced the CCD array by a $1 \mathrm{GHz}$ avalanche photodetector (APD; Menlo Systems APD210) synchronized with the LD laser through a highperformance serial bit error ratio (BER) tester (Agilent Technologies J-BERT N4903B). A graph of the BER performance for different system data rates is depicted in Fig. 2(c). An error-free link is achieved up to 1.06 Gbit/s, as well as a maximum data rate of $1.80 \mathrm{Gbit} / \mathrm{s}$ with BER of $0.4 \times 10^{-3}$ below the forward error correction (FEC) limit $3.8 \times 10^{-3}$. We should stress that the limiting factor of the bit rate is the 3 -dB bandwidth of the APD that falls right at $1 \mathrm{GHz}$. Thus, the fabricated SPPs can be used to generate OAM beams able to carry high bit rate data signals.

\section{Conclusion}

In summary, we used $20 \mu \mathrm{m}$ diameter SPPs to generate pure OAM beams used to establish free space communication links. Such SPPs could be integrated into lasers and photonic circuits. We believe that our demonstrations form a first step towards cost-effective high-speed OAM-based integrated communication systems.

\section{Funding}

King Abdullah University of Science \& Technology BAS/1/1614-01-01, KCR/1/2081-01-01, GEN/1/6607-01-01.

\section{References}

1. G. Gibson et al., "Free-space information transfer using light beams carrying orbital angular momentum," Opt. Express 12, 5448-5456, 2004.

2. A. E. Willner et al., "Optical communications using orbital angular momentum beams," Adv. Opt. Photon. 7, 66-106, 2015.

3. S. Yu, "Potentials and challenges of using orbital angular momentum communications in optical interconnects," Opt. Express 23, 3075-3087, 2015.

4. I. A. Litvin, A. Dudley, F. S. Roux, and A. Forbes, "Azimuthal decomposition with digital holograms," Opt. Express 20, 10996-11004, 2012.

5. V. Arrizón, U. Ruiz, R. Carrada, and Luis A. González, "Pixelated phase computer holograms for the accurate encoding of scalar complex fields,” J. Opt. Soc. Am. A 24, 3500-3507, 2007. 